\title{
MITTEILUNGEN DER OBVSG
}

\section{von Mathis Kronschläger}

Zusammenfassung: In den „Mitteilungen der OBVSG“ gibt die Österreichische Bibliothekenverbund und Service $\mathrm{GmbH}$ einen Überblick über aktuelle Ereignisse aus dem Verbund und der Verbundzentrale.

Schlagwörter: OBVSG; Alma; Primo; Primo New Userinterface

\section{COMMUNICATIONS OF THE AUSTRIAN LIBRARY NETWORK AND SERVICES LTD (OBVSG)}

Abstract: The Austrian Library Network and Services Ltd (OBVSG) provides services for Austrian research and administrative libraries and is the library network's headquarter. „Communications of OBVSG" informs about current and upcoming events concerning OBVSG and the Austrian Library Network.

Keywords: Austrian Library Network and Services Ltd (OBVSG); Alma; Primo; Primo New Userinterface

\section{Inhalt}

1. Go-live der Wave 4

2. Bibliothek des Bundesministeriums für Finanzen und Wienbibliothek im Rathaus neue Primo-Teilnehmer

3. Sieben weitere Bibliotheken mit neuem Primo Userinterface online

4. Kick-off der Wave 5

DOI: https://doi.org/10.31263/voebm.v72i1.2283

(C) Mathis Kronschläger

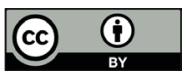

Dieses Werk ist lizenziert unter einer

Creative-Commons-Lizenz Namensnennung 4.0 International 


\section{Go-live der Wave 4}

Seit 16. Jänner sind sieben weitere Bibliotheken des OBV live mit Alma!

Die Bibliotheken dieser Einrichtungen bilden gemeinsam die Wave 4:

- Bundesministerium für Finanzen

- Internationale Stiftung Mozarteum

- Landesmuseum Kärnten - Kärntner Landesbibliothek

- MAK - Österreichisches Museum für angewandte Kunst /

Gegenwartskunst

- Max Perutz Library

- Österreichische Akademie der Wissenschaften

- Österreichisches Museum für Volkskunde

Mit dem Go-live ist die Alma-Implementierung beinahe abgeschlossen. Auf die Migration folgt eine Nachbereitungszeit, in der letzte Einstellungen vorgenommen werden. Sobald diese Arbeiten getan sind, ist das Projektziel erreicht und der reguläre Betrieb kann aufgenommen werden.

Die Wave 4 brachte für das Team der OBVSG einiges Neues. Zum ersten Mal wechselten - nunmehr ehemalige - Aleph-Sharing-Bibliotheken auf Alma. Für die OBVSG stellte die Migration dieser sechs Bibliotheken eine neue Herausforderung dar, denn sie ist bei ihnen für sämtliche systembibliothekarische Aufgaben verantwortlich.

Ebenfalls neu seit der Wave 4 ist, dass Schulungen vom Team der OBVSG durchgeführt werden. Teilnehmen können sowohl Bibliotheken, die von der OBVSG systembibliothekarisch betreut werden, als auch solche, die diese Aufgaben selbst ausführen. Wie bei der Einführung von RDA in den Bibliothekenverbund wird wieder auf ein Multiplikatoren-System gesetzt. Vertreterinnen und Vertreter der Bibliotheken nehmen an den Schulungen teil und geben ihr Wissen anschließend in ihren Bibliotheken weiter. Bei den Schulungen angeboten werden Einheiten zu Katalogisierung und MARC 21, Ausleihe, Erwerbung und Zeitschriftenverwaltung.

Nach dem Go-live ist vor dem Go-live: Die Bibliotheken der Wave 5 sind bereits in der Onboarding-Phase. Ihr Kick-off wird im Februar stattfinden.

\section{Bibliothek des Bundesministeriums für Finanzen und Wienbibliothek im Rathaus neue Primo-Teilnehmer}

Wir freuen uns, dass sich zwei weitere Bibliotheken des OBV für die Suchmaschine Primo entschieden haben. 
Beide Einrichtungen nehmen an der konsortialen Primo-Instanz im Modell Primo Flex teil. In diesem Modell richtet das Primo-Team der OBVSG die Suchmaschine in enger Absprache mit den Bibliotheken ein und übernimmt alle anfallenden Service-Aufgaben des laufenden Betriebs.

Der Go-live mit Primo steht für die Bibliothek des Bundesministeriums für Finanzen unmittelbar bevor. Sie wird von Anfang an das neue Primo Userinterface verwenden. Erst vor wenigen Tagen ist sie als Einrichtung der Wave 4 mit Alma in Produktion gegangen.

Die Wienbibliothek im Rathaus ist seit Anfang Dezember live mit Primo. Die Übernahme der Sammlungsbereiche und Funktionalitäten aus dem OPAC waren zum Teil eine große Herausforderung, konnten aber nach intensiver Zusammenarbeit mit dem Team der Wienbibliothek gut umgesetzt werden. Der Wechsel auf das neue Userinterface folgt für die Wienbibliothek in einem nächsten Schritt. Auch sie wird den Umstieg auf Alma für den Wechsel auf das neue Userinterface nutzen.

\section{Sieben weitere Bibliotheken mit neuem Primo Userinterface online}

In den letzten beiden Monaten hat sich viel getan bei Primo!

Immer mehr Bibliotheken entscheiden sich für das neue Userinterface, das sich nun optimal an das Display anpasst, gleich ob es sich um Smartphone, Tablet oder Desktop-Computer handelt.

Sechs Bibliotheken der Wave 4 nutzten die Gelegenheit des AlmaUmstiegs, um das neue Primo Userinterface zu implementieren. Nach dem Go-live mit Alma Mitte Jänner sind sie jetzt mit der neuen Bibliothekssuchmaschine online.

- Bundesministerium für Finanzen

- Internationale Stiftung Mozarteum

- Landesmuseum Kärnten - Kärntner Landesbibliothek

- MAK - Österreichisches Museum für angewandte Kunst / Gegenwartskunst

- Max Perutz Library

- Österreichisches Museum für Volkskunde

Auch die Veterinärmedizinische Universität Wien ist bereits auf das neue Userinterface umgestiegen. Sie ist mit Anfang Dezember in Produktion gegangen.

Damit sind es bereits 12 Bibliotheken im OBV, die das neue Primo Userinterface im responsiven Webdesign nutzen. Weitere folgen in Kürze. 


\section{Kick-off der Wave 5}

Mit dem Kick-off-Meeting am 12. Februar machen sich acht weitere Bibliotheken auf den Weg nach Alma.

Beim Onboarding im Herbst wurden jene Aufgaben besprochen, welche bereits im Vorfeld der Migration angegangen werden können. Nun beginnt mit dem Kick-off die eigentliche Alma-Implementierung. Ein voller Tag steht Ex Libris, der OBVSG und den Bibliotheken der Wave 5 zur Verfügung, um den genauen Ablauf der nächsten Monate zu besprechen. Zusätzlich zu den Präsentationen und Fragerunden im Vortragssaal besteht die Möglichkeit, Details des Systemwechsels in Einzelgesprächen zu klären.

\section{Von Aleph-Sharing nach Alma}

Sechs der acht Bibliotheken wechseln vom Modell Aleph-Sharing nach Alma. Bei diesen Bibliotheken übernimmt das systembibliothekarische Team der OBVSG die Migration des Systems, wobei besonders der umfangreiche Entlehnbetrieb einiger Einrichtungen viel Neues mit sich bringt. Auch das Primo-Team der OBVSG ist bei der Wave 5 erneut gefordert. Für ganze sieben Einrichtungen wird Primo auf Alma angepasst, Umstellung auf das neue Userinterface inklusive.

\section{Die Bibliotheken der Wave 5 im Überblick}

- Akademie der bildenden Künste Wien

- Donau-Universität Krems

- Fachhochschule Salzburg

- FH Campus Wien

- FH Campus 02 Fachhochschule der Wirtschaft

- FHWien - Studiengänge der Wirtschaftskammer Wien

- Oberösterreichische Landeskultureinrichtungen

- Paracelsus Medizinische Privatuniversität

Die Akademie der bildenden Künste Wien und die Oberösterreichischen Landeskultureinrichtungen übernehmen mit eigenem Personal die systembibliothekarischen Aufgaben des Systemwechsels. Erstere setzt auf das Discovery System Summon, zweitere implementieren gemeinsam mit der OBVSG Primo für Alma. 
Mag. Mathis Kronschläger Die Österreichische Bibliothekenverbund und Service GmbH (OBVSG) E-Mail: mathis.kronschlaeger@obvsg.at 